\title{
Several Notations of Gronwall Inequality of Vector Functions
}

\author{
D.Luo ${ }^{1,2, a}$, Y.Liu $^{1, b}$, C.Wang ${ }^{1, c}$
}

${ }^{1}$ The school of Mathematics of Zunyi Normal college,Zunyi, GuizhouProvince,563002,P.R. China adsluo99@126.com, ${ }^{\text {b5 } 546692653 @ q q . c o m,{ }^{c} 2941359790 @ q q . c o m, ~}$

Keywords: Gronwell-inequality,vector function inequality, Holder inequality, Minkoviski inequality, Maximum principle

Abstract In this paper, we first consider tow inequalities of vector functions,then combine the vector function inequality with Gronwell' inequality to obtain another two vector function's inequalities. At last, using them to prove the maximum principle for one kind of optimal control problem.

\section{Introduction and derivation of vector funtions' inequality}

Gronwell inequalities show the relationship among functions and their derivatives and integration[1], and are widely used to estimate functions' value in function spaces such as $L^{P}$ space so on.They are very important for embedding theories of Sobolev space[2,3], also are key tools for research on the solution of $\mathrm{PDE}[4]$ and some problems of optimal control of differential equation[5,6,7].For convenience, we first list the two lemmas bellowing:

Lemma.1 [8]Let $y(t)$ be a continuously differential vector value function on the interval $\left[t_{0}, t_{1}\right]$, then $\frac{d}{d t}\|y(t)\| \leq\left\|\frac{d}{d t} y(t)\right\|$,for all $t \in\left[t_{0}, t_{1}\right]$, and $y(t) \neq 0$.

Lemma.2.[9] Let $y(t)$ be continuous vector valued function on $\left[t_{0}, t_{1}\right]$.If $y(t)$

satisfies $\|y(t)\| \leq a \varepsilon+\beta \int_{t_{1}}^{t}\|y(\delta)\| d \delta, \forall t \in\left[t_{1}, t_{2}\right]$ for given positive constant $a, \beta, \varepsilon$, then $\|y(t)\| \leq a \varepsilon e^{\beta\left(t-t_{1}\right)}, \forall t \in\left[t_{1}, t_{2}\right]$.

Then show a vector function inequality from Gronwell inequality.

Proposition.3.Let $y(t)$ be continuously differential vector-valued function on the interval $\left[t_{0}, t_{1}\right]$, and $y_{i}^{\prime}(t) \leq \phi_{i}(t) y_{i}(t)+\psi_{i}(t), i=1,2, \cdots n$, then

$$
\|y(t)\| \leq e^{\int_{t_{0}}^{t}\|\phi(s)\| d s}\left[\left\|y\left(t_{0}\right)\right\|+\int_{t_{0}}^{t}\|\psi(s)\| d s\right]
$$

Where $y(t)=\left(y_{1}(t), \cdots y_{n}(t)\right), \phi(t)=\left(\phi(t), \phi_{2}(t) \cdots \phi_{n}\right), \psi(t)=\left(\psi_{1}(t), \psi_{2}(t), \cdots \psi_{n}(t)\right)$ and $\|y(t)\|,\|\phi(t)\|,\|\psi(t)\|$ are the Euclidean norms correspondingly.

Proof. From lemma.1., Minkowski inequality and Hölder inequality , we can obtain

$$
\|y(t)\| \leq\|\phi(t)\|\|y(t)\|+\|\psi(t)\| .
$$

Hence through the differential form Gronwell inequality, we obtain

$$
\|y(t)\| \leq e^{\int_{t_{0}}^{t}\|\phi(s)\| d s}\left[\left\|y\left(t_{0}\right)\right\|+\int_{t_{0}}^{t}\|\psi(s)\| d s\right] .
$$

Proposition.4. Let $y(t)$ be continuous vector function on the interval $\left[t_{0}, t_{1}\right]$ If $y(t)$ satisfies $\|y(t)\| \leq a \varepsilon+\beta \int_{t_{1}}^{t}\|y(\delta)\| d \delta, \forall t \in\left[t_{1}, t_{2}\right]$ for given positive constant $a, \beta, \varepsilon$. Then $\|y(t)\| \leq a \varepsilon\left[1+\beta\left(t-t_{0}\right) e^{\beta\left(t-t_{0}\right)}\right]$. 
The proof is trivial with integral form Gronwell inequality.

\section{An Example of the Inequalities' Application}

Example. Consider the optimal control problem which system is stated by $x=f(x, u), x\left(t_{0}\right)=x_{0}$, where $x \in R^{n}$, control variable $u \in R^{r}$, the objective set is $x\left(t_{f}\right) \in R^{n}$ and $t_{f}$ is fixed. $f: R^{n} \times R^{r} \rightarrow R^{n}$ is $C^{1}, U_{a d}=\left\{u(t) \mid u(t) \in U_{r} \subseteq R^{r}\right\}$,

where $u(t)$ is piece-wise continuous functions and $U_{r} \in R^{r}$ is bounded closed set, $x(t)$ is the state variable corresponding to the control variable $u(\cdot)$ and its initial state satisfies $x\left(t_{0}\right)=x_{0}$, set the cost functional as following

$$
J[u(\cdot)]=K\left(x\left(t_{f}\right)\right)+\int_{t_{0}}^{t_{f}} L(x, u) d t .
$$

To ensure the existence of the solution of the optimal control problem, we assume that

(i) $f(x, u), L(x, u), K(x)$ are continuous for their variable and are continuously differential to the variable $x$;(ii) $f(x, u), \frac{\partial f(x, u)}{\partial x}, \frac{\partial L(x, u)}{\partial x}$ all are bounded.

Since $f(\bar{x}, u)-f(\hat{x}, u)=\int_{\hat{x}}^{-} \frac{\partial f(x, u)}{\partial x} d x$, there are two positive constants $a, b$ which are independent to $x, u$ such that $\|f(x, u)\| \leq \frac{a}{2}, \forall u \in U, x \in R^{n},\|f(\bar{x}, u)-f(\hat{x}, u)\| \leq b\|\hat{x}-\hat{x}\|$, where $\|\cdot\|$ is the norm of Euchiled vector.

Proposition5 if $u^{*}(t)$ is the optimal control of the above problem, the necessary condition is that $H\left(x^{*}(t), u^{*}(t), \psi(t)\right)=\max _{\substack{v \\ u \in U_{r}}} H\left(x^{*}(t), u(t), \psi(t)\right)$.

Proof. Let $\psi(t) \in R^{n}$,then problem (4) can be transformed to the problem

$$
J\left[u(\cdot]=K\left(x\left(t_{f}\right)\right)+\psi^{T}\left(t_{f}\right) x\left(t_{f}\right)-\psi^{T}(0) x_{0}-\int_{t_{0}}^{t_{f}}[\dot{\psi}(t) x(t)+H(x(t), u(t), \psi(t))] d t\right.
$$

without constrain condition and where

$H(x, u, \psi)=-L(x, u)+\psi^{T} f(x, u)$ is the Hamilton function,assume $\left(u^{*}(t), x^{*}(t)\right)$ be the optimal pairs of the optimal control problem. Given

$$
u(t)=u^{*}(t)+\Delta u(t), t \in\left[t_{0}, t_{f}\right]
$$

to ensure $u(t) \in U_{\left[t_{0}, t_{f}\right]}$, then $\Delta u(t)$ must satisfies two conditions : $\Delta u(t)$ define on $\left[t_{0}, t_{f}\right]$, and are continuous vector functions, $u(t)=u^{*}(t)+\Delta u(t) \in U_{r}, t \in\left[t_{0}, t_{f}\right]$.

And $x(t)=x^{*}(t)+\Delta x(t), t \in\left[t_{0}, t_{f}\right]$ is the solution of optimal control problem corresponding to $u(t)$ above. It is trivial that $\Delta x(t)$ satisfies

$$
\left\{\begin{array}{l}
\frac{d}{d t} \Delta x(t)=f\left(x^{*}(t)+\Delta x(t), u^{*}(t)+\Delta u(t)\right)-f\left(x^{*}(t), u^{*}(t)\right), \\
\Delta x\left(t_{0}\right)=0
\end{array}\right.
$$

Then we obtain

$$
\Delta J\left[u^{*}(\cdot)\right] \triangleq J[u(\cdot)]-J\left[u^{*}(\cdot)\right] \quad=\mathrm{K}\left(\mathrm{x}^{*}\left(t_{f}\right)+\Delta x^{*}\left(t_{f}\right)\right)-K\left(x^{*}\left(t_{f}\right)+\psi^{T}\left(t_{f}\right) \Delta x\left(t_{f}\right)\right.
$$


$-\int_{t_{0}}^{t_{f}} \psi(t) \Delta x(t) d t-\int_{t_{0}}^{t_{f}}\left[H\left(x^{*}(t)+\Delta x(t), u^{*}(t)+\Delta u(t), \psi(t)\right)-H\left(x^{*}(t), u^{*}(t), \psi(t)\right)\right] d t$.

And according to the assumptions of $f(x, u), L(x, u), K(x)$, we get

$$
\begin{gathered}
K\left(x^{*}\left(t_{f}\right)+\Delta x\left(t_{f}\right)\right)-K\left(x^{*}\left(t_{f}\right)\right)=\frac{\partial K\left(x^{*}\left(t_{f}\right)\right)}{\partial x} \Delta x\left(t_{f}\right)+o\left(\left\|\Delta x\left(t_{f}\right)\right\|\right), \\
\Delta H\left(x^{*}(t), u^{*}(t), \psi(t)\right)=H\left(x^{*}(t)+\Delta x(t), u^{*}(t)+\Delta u(t), \psi(t)\right)-H\left(x^{*}, u^{*}, \psi(t)\right) \\
=\left\{\frac{\partial F\left(x^{*}(t), u^{*}(t)+\Delta u(t), \psi(t)\right)}{\partial x}+\frac{\partial H\left(x^{*}(t), u^{*}(t), \psi(t)\right)}{\partial x}\right\} \Delta x(t) \\
+o(\|\Delta x(t)\|)+F\left(x^{*}(t), u^{*}(t)+\Delta u(t), u^{*}(t), \psi(t)\right) .
\end{gathered}
$$

where $F\left(x^{*}(t), u^{*}(t)+\Delta u(t), u^{*}(t), \psi(t)\right)=\mathrm{H}\left(\mathrm{x}^{*}(t), u^{*}(t)+\Delta u(t), \psi(t)\right)-H\left(x^{*}(t), u^{*}(t), \psi(t)\right)$ then $\Delta J\left[u^{*}(\cdot)\right]=\left[\frac{\partial K\left(x^{*}\left(t_{f}\right)\right.}{\partial x}+\psi^{T}\left(t_{f}\right)\right] \Delta x\left(t_{f}\right)-\int_{t_{0}}^{t_{f}}\left[\psi(t)+\left.\frac{\partial H}{\partial x}\right|_{*}\right] \Delta x(t) d t$

$$
\begin{aligned}
& -\int_{t_{0}}^{t_{f}} \frac{\partial F\left(x^{*}(t), u^{*}(t)+\Delta u(t), u^{*}(t), \psi(t)\right)}{\partial x} \Delta x(t) d t \\
& -\int_{t_{0}}^{t_{f}} F\left(x^{*}(t), u^{*}(t)+\Delta u(t), u^{*}(t), \psi(t)\right) d t+\int_{t_{0}}^{t_{f}} o(\|\Delta x(t)\|) d t+o\left(\left\|\Delta x\left(t_{f}\right)\right\|\right),
\end{aligned}
$$

where

$$
\left.\frac{\partial H}{\partial x}\right|_{*}=\frac{\partial H\left(x^{*}(t), u^{*}(t), \psi(t)\right)}{\partial x} .
$$

As we know that $\psi(t)$ satisfies the condition below:

Then

$$
\left\{\begin{array}{l}
\psi^{\mathrm{T}}(t)=-\frac{\partial H\left(x^{*}(t), u^{*}(t), \psi(t)\right)}{\partial x} \\
\psi^{T}\left(t_{f}\right)=-\frac{\partial K\left(x^{*}\left(t_{f}\right)\right)}{\partial x}
\end{array}\right.
$$

$$
\begin{aligned}
& \Delta J\left[u^{*}(\cdot)\right]=-\int_{t_{0}}^{t_{f}} \frac{\partial F\left(x^{*}(t), u^{*}(t)+\Delta u(t), u^{*}(t), \psi(t)\right)}{\partial x} \Delta x(t) d t \\
& \quad-\int_{t_{0}}^{t_{f}} F\left(x^{*}(t), u^{*}(t)+\Delta u(t), u^{*}(t), \psi(t)\right) d t+\int_{t_{0}}^{t_{f}} o(\|\Delta x(t)\|) d t+o\left(\left\|\Delta x\left(t_{f}\right)\right\|\right) \mathrm{d} t .
\end{aligned}
$$

As we know that for any $\Delta u(t)$ of $u^{*}(t),(9)$ hold. So when we chose a special

$$
u(t)=\left\{\begin{array}{l}
u^{*}(t), t \in\left[t_{0}, \bar{t}\right) \cup\left[\bar{t}+\varepsilon, t_{f}\right] \\
\hat{u}, \quad t \in[\bar{t}, \bar{t}+\varepsilon)
\end{array}\right.
$$

where $\varepsilon$ is any sufficient small positive real number,and $\hat{u} \in U_{r}$ is any $r$-dimension constant vector, setting $\Delta_{\varepsilon t} u(t)=\left\{\begin{array}{l}0, \quad t \in\left[t_{0}, \bar{t}\right) \cup\left[\bar{t}+\varepsilon, t_{f}\right] \\ \hat{u}-u^{*}(t) \quad t \in[\bar{t}, \bar{t}+\varepsilon)\end{array}\right.$

If $\Delta_{\varepsilon t} x(t), \Delta_{\varepsilon t} J\left[u^{*}(\cdot)\right]$ are the change quantity of $x^{*}, J\left[u^{*}(\cdot)\right]$ corresponding to $\Delta_{\varepsilon t} u(t)$, then from(6), $\Delta_{\varepsilon t} x(t)$ will result

$$
\Delta_{\varepsilon t} x(t)=0, \quad t \in\left[t_{0}, \bar{t}\right],
$$


$\left\{\begin{array}{l}\frac{d}{d t} \Delta_{\varepsilon \bar{t}} x(t)=f\left(x^{*}(t)+\Delta_{\varepsilon t} x(t), \hat{u}(t)\right)-f\left(x^{*}(t), u^{*}(t)\right), \quad t \in\left[\bar{t}, t_{f}\right], \\ \Delta_{\varepsilon t} x(\bar{t})=0, \quad t \in[\bar{t}, t+\varepsilon), \\ \Delta_{\varepsilon t}^{-} x(\bar{t}+\varepsilon)=\text { initiable value, } \quad t \in\left[\bar{t}+\varepsilon, t_{f}\right]\end{array}\right.$,

Therefore

$$
\begin{aligned}
\Delta_{\varepsilon t} J[ & \left.u^{*}(\cdot)\right]=-\int_{t}^{-t+\varepsilon} \frac{\partial F\left(x^{*}(t), \hat{u}(t), u^{*}(t), \psi(t)\right)_{-}}{\partial x} \Delta_{\varepsilon t} x(t) d t \\
& \quad-\int_{t}^{-} F\left(x^{-}(t), \hat{u}, u^{*}(t), \psi(t)\right) d t+\int_{t_{0}}^{t_{f}} o\left(\left\|\Delta_{\varepsilon t} x(t)\right\|\right) d t+o\left(\left\|\Delta_{\varepsilon t} x\left(t_{f}\right)\right\|\right) .
\end{aligned}
$$

As $t \in[\bar{t}, \bar{t}+\varepsilon]$, note (4) and Lemma.1, and from (13) we can obtain directly that

$$
\frac{d}{d t}\left\|\Delta_{\varepsilon t} x(t)\right\| \leq\left\|\frac{d}{d t} \Delta_{\varepsilon t} x(t)\right\| \leq a+b\left\|\Delta_{\varepsilon t} x(t)\right\|
$$

Since $\Delta_{\varepsilon t} x(\bar{t})=0$, then integration both sides above formula from $\bar{t}$ to $t$

$$
\left\|\Delta_{\varepsilon t} x(t)\right\| \leq a\left(t-t_{f}\right)+b \int_{t_{f}}^{t}\left\|\Delta_{\varepsilon t} x(\sigma)\right\| d \sigma \leq a \varepsilon+\int_{t_{f}}^{t}\left\|\Delta_{\varepsilon t} x(\sigma)\right\| d \sigma
$$

and with the Lemma.2., we get

$$
\left\|\Delta_{\varepsilon t} x(t)\right\| \leq a \varepsilon e^{b\left(t-t_{f}\right)} \leq a \varepsilon e^{b \varepsilon}=\mathrm{O}(\varepsilon), \quad t \in[\bar{t}, \bar{t}+\varepsilon] .
$$

And (14)implies that $\left\|\Delta_{\varepsilon t} x(t)\right\|$ is at most the same order as $\varepsilon$ on the interval $[\bar{t}, \bar{t}+\varepsilon]$. As $t \in[\bar{t}, \bar{t}+\varepsilon]$, through Lemma1.and (14), we can directly get

$$
\frac{d}{d t}\left\|\Delta_{\varepsilon \bar{t}} x(t)\right\| \leq\left\|\frac{d}{d t} \Delta_{\varepsilon \bar{t}} x(t)\right\| \leq b\left\|\Delta_{\varepsilon \bar{t}} x(t)\right\| \text {, }
$$

then integrate both sides of the inequality from $\bar{t}+\varepsilon$ to $t$, and get $\left\|\Delta_{\varepsilon t} x(t)\right\| \leq\left\|\Delta_{\varepsilon t} x(\bar{t}+\varepsilon)\right\|+b \int_{t}^{t}\left\|\Delta_{\varepsilon t}^{t} x(\sigma)\right\| d \sigma$. According to Lemma.2., we get

$$
\left.\left\|\Delta_{\varepsilon t} x(t)\right\| \leq\left\|\Delta_{\varepsilon t} x \overline{(t+\varepsilon)}\right\| e^{b[t-\bar{t}+t)]} \leq\left\|\Delta_{\varepsilon t}^{-x} \overline{(t+\varepsilon)}\right\| e^{b\left[t_{f}-\overline{(t+t)]}\right.} \leq \mathrm{O}(\varepsilon), \forall t \in \bar{t} \bar{t}+\varepsilon, t_{f}\right] .
$$

Combination with(11),(12),(14)and(15), we gain $\left\|\Delta_{\varepsilon t} x(t)\right\| \leq \mathrm{O}(\varepsilon), \forall t \in\left[t_{0}, t_{f}\right]$, and submit it to (14), then $\Delta_{\varepsilon t} J\left[u^{*}(\cdot)\right]=-\int_{t}^{\bar{t}+\varepsilon} F\left(x^{*}(t), \hat{u}(t), u^{*}(t), \psi(t)\right) d t+o(\varepsilon)$ Since $F(\cdot, \cdot, \cdot, \cdot)$ is continuous and $u^{*}(t)$ is continuous at $\bar{t}$, from the above we can get $\Delta_{\varepsilon t} J\left[u^{*}(\cdot)\right]=-\varepsilon F\left(x^{*}(\bar{t}), \hat{u}(t), u^{*}(\bar{t}), \psi(\bar{t})\right)+o(\varepsilon)=-\varepsilon\left[H\left(x^{*}(\dot{t}), \hat{u}, \psi(\bar{t})\right)-H\left(x^{*}(\bar{t}), u^{*}(\bar{t}), \psi(\bar{t})\right)\right]+o(\varepsilon)$. And $u^{*}(t)$ is the optimal control,therefore, for any $\Delta_{\varepsilon t} u(t)$ as the form of (10), $\Delta_{\varepsilon t} J\left[u^{*}(\cdot)\right] \geq 0$, namely, $-\varepsilon\left[H\left(x^{*}(\bar{t}), \hat{u}, \psi(\bar{t})\right)-H\left(x^{*}(\bar{t}), u^{*}(\bar{t}), \bar{\psi}(\bar{t})\right)\right]+o(\varepsilon) \geq 0$, 
and $\lim _{\varepsilon \rightarrow 0} \frac{o(\varepsilon)}{\varepsilon}=0$. Hence $H\left(x^{*}(\bar{t}), \hat{u}, \psi(\bar{t})\right) \leq H\left(x^{*}(\bar{t}), u^{*}(\bar{t}), \bar{\psi}(\bar{t})\right)$. Since $\hat{u} \in U_{r} \quad$ is $\quad$ any $r$-dimension vector and $u^{*}(t)$ is continuous on the interval $\left[t_{0}, t_{f}\right]$, therefore, $H\left(x^{*}(t), u^{*}(t), \psi(t)\right)=\max _{\substack{v \\ u \in U_{r}}} H\left(x^{*}(t), u(t), \psi(t)\right)$, and the proposition holds.

\section{Acknowledgement}

In this paper, the research was supported by the National Nature Science Foundation of China (Grants no. 71461027, 71471158);Science and technology of

Guizhou province outstanding youth (Qian ke he ren zi [2015]06);Guizhou science and technology cooperation plan (Qian Ke He LH zi [2015]7004,[2016]7028,7031).

\section{References}

[1] Zhao Yun. A Note on Gronwall Inequality. Studies in College Mathematics,

a) Vol.14,No.4,Jul(2011)14- 18.

[2] Eberhard Zeidler. Nonlinear Functional Analysis and its Applications 2/A:Linear Monotone Operators.Springer-Verlag-Newyork,1990.

[3] Eberhard Zeidler. Nonlinear Functional Analysis and its Applications

a) 2/B:Nonlinear Monotone Operators.Springer-Verlag,Newyork,1990.

[4] C.L.Evans. Partial Differential Equations.the American Mathematical Society,2002.

[5] J. Arthur,Krener,Wei Kang. Linar Time Invariant Minimax Filtering. Annual Reviews in Control 35(2011)166-171.

[6] M.Athans,P.L.Falb. Optimal Control:An Introduction to the Theory and its Application.NewYork:McGraw-Hill,1996.

[7] B.D. Anderson,J.,B.Moore. Linear Optimal Control. Prentice-Hall,1971.

[8] A.J.Sorensen. A Survey of Dynamic Positioning Control System. Annual Reviews in Con- trol 35(2011)123-136.

[9] Lubomír Bakule Martin Papík. Decentralized Control and Communication. Annual Reviews in Control 36(2012)1-10. 